\title{
Peritectic Formation and Phase Stability of the Icosahedral Quasicystal i-GdCd and Its Ternary Variants with Zn, Mg, and Y
}

\author{
Girma Gebresenbut,* Daniel Eklöf, Alisa Gordeeva, Takayuki Shiino, and Ulrich Häussermann
}

Cite This: Cryst. Growth Des. 2021, 21, 3355-3363

Read Online
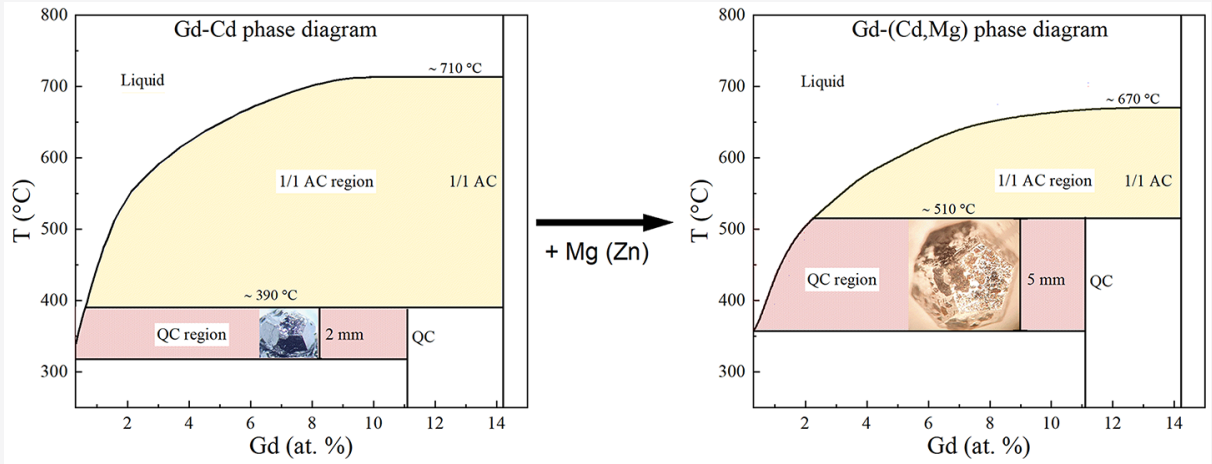

ABSTRACT: Stable binary icosahedral quasicrystals (i-QCs) based on rare earth (RE) and cadmium are typically accessed by solution growth experiments, which operate in very narrow composition and temperature windows. Here, we present a procedure which allows study of peritectic reactions between approximant crystal (AC) phase and liquid yielding i-RECd and exemplify with i$\mathrm{GdCd}$ and ternary variants where $\mathrm{Cd}$ is partially replaced by isovalent $\mathrm{Zn}(\mathrm{i}-\mathrm{Gd}(\mathrm{Cd}, \mathrm{Zn})$ ) or $\mathrm{Mg}(\mathrm{i}-\mathrm{Gd}(\mathrm{Cd}, \mathrm{Mg})$ ), or the $4 \mathrm{f}$ element $\mathrm{Gd}$ is replaced by nonmagnetic $\mathrm{Y}(\mathrm{i}-(\mathrm{Gd}, \mathrm{Y}) \mathrm{Cd})$. The solubility limits for $\mathrm{Zn}$ and $\mathrm{Mg}$ substitution are about $10 \%$ and $20 \%$, respectively, whereas Gd and $\mathrm{Y}$ show a complete solid solution behavior. We find that the peritectic decomposition temperature for $\mathrm{i}-\mathrm{GdCd}$ is $390{ }^{\circ} \mathrm{C}$, which is decreased when $\mathrm{Gd}$ is replaced by $\mathrm{Y}\left(\mathrm{i}-\mathrm{YCd}: 350{ }^{\circ} \mathrm{C}\right.$ ) and increased when Cd is replaced by $\mathrm{Zn}(\mathrm{i}-$ $\left.\mathrm{Gd}\left(\mathrm{Cd}_{90} \mathrm{Zn}_{10}\right): 440{ }^{\circ} \mathrm{C}\right)$, and especially by $\mathrm{Mg}\left(\mathrm{i}-\mathrm{Gd}\left(\mathrm{Cd}_{80} \mathrm{Mg}_{20}\right): 520^{\circ} \mathrm{C}\right)$. Whereas substitution decisively alters the decomposition temperature (and hence stability) of the considered i-QCs, the decomposition temperature of the corresponding $\mathrm{AC}$ phases remains at around $700{ }^{\circ} \mathrm{C}$. During the investigation of the pseudobinary phase diagrams Gd- $\left(\mathrm{Cd}_{95} \mathrm{Zn}_{5}\right), \mathrm{Gd}-\left(\mathrm{Cd}_{90} \mathrm{Zn}_{10}\right)$, and Gd- $\left(\mathrm{Cd}_{80} \mathrm{Mg} \mathrm{g}_{20}\right)$, faceted i-QCs grains with sizes up to $4 \times 4 \times 4 \mathrm{~mm}^{3}$ could be isolated.

\section{INTRODUCTION}

Quasicrystals (QCs) typically are intermetallic compounds which realize long-range positional order without imposing periodicity in three dimensions. ${ }^{1}$ The formation of QCs and their stability with respect to $3 \mathrm{D}$ periodic and compositionally similar approximant crystals (ACs) has been intensely researched, but is still puzzling. ${ }^{2-8}$ The discovery of the icosahedral QCs (i-QCs) $\mathrm{Cd}-\mathrm{Yb}$ and $\mathrm{Cd}-\mathrm{Ca}$ by Tsai et al. $(2000)^{3,9}$ represented an important milestone in that it showed that intermetallic QCs can represent thermodynamically stable binary phases. Today, Tsai-type i-QCs are recognized as line phases in a number of binary alkaline earth and rare earth (RE) $\mathrm{Cd}$ and $\mathrm{Zn}$ phase diagrams. ${ }^{10,11}$ Yet, only prototypic i-YbCd $\left(\mathrm{YbCd}_{5.7}\right)$ represents a congruently melting phase. Others are well hidden in their phase diagrams and only accessible in small composition-temperature windows. Competing phases are cubic AC phases with very similar composition, e.g., $\mathrm{RECd}_{\sim 6}$ $(1 / 1)$ and $\operatorname{RECd}_{\sim 5.8}(2 / 1)$, as well as more Cd- or Zn-rich phases with stoichiometries $1: 11,1: 12$, or $1: 13$.
Particularly successful has been the growth of binary QCs from Cd- or Zn-rich melts (solution growth experiments) upon finding appropriate composition-temperature windows, i.e., the region of exposed liquidus line. ${ }^{10,12}$ This is sketched in Figure 1 with the phase diagrams of $\mathrm{Gd}-\mathrm{Cd}$ and $\mathrm{Sc}-\mathrm{Zn}$. In the following, we refer to this as "High Melt Crystallization (HMC)". On the other hand, if the binary QC represents a stable phase, it should also be accessible through peritectic reactions of AC phase with either $\mathrm{Cd} / \mathrm{Zn}$-rich liquid or more $\mathrm{Cd} / \mathrm{Zn}$-rich phase, if present. This, however, has been rarely observed, and as a consequence, peritectic decomposition temperatures of binary QCs-which can be considered as indicators for QC stability-are largely unknown. A reason could be that, in contrast with HMC,

Received: February 5, 2021

Revised: April 27, 2021

Published: May 7, 2021 

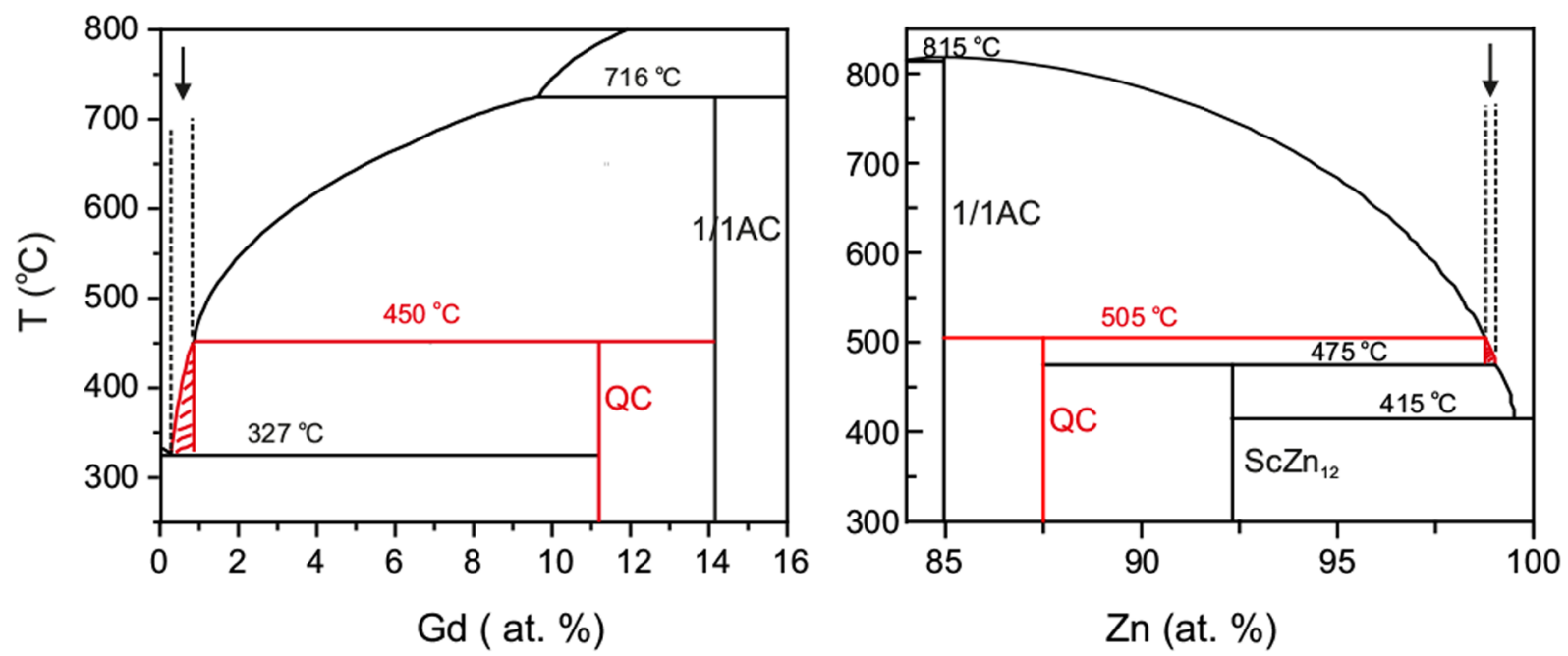

Figure 1. Partial-phase diagram of $\mathrm{Gd}-\mathrm{Cd}(\mathrm{left})^{11}$ and $\mathrm{Sc}-\mathrm{Zn}$ (right). ${ }^{10}$ The exposed solidus line and composition-temperature window for accessing the QC phase by "high melt crystallization" (HCM) are indicated.

Table 1. Synthesis Conditions Used to Prepare QCs and 1/1 ACs Using the HMC and LMPF Synthesis Protocols ${ }^{a}$

\begin{tabular}{|c|c|c|c|c|c|c|}
\hline system & $\begin{array}{l}\text { starting nominal } \\
\text { composition }\end{array}$ & $\begin{array}{l}\text { synthesis } \\
\text { method }\end{array}$ & $\begin{array}{l}\text { resulting } \\
\text { phase }\end{array}$ & $\begin{array}{l}\text { starting total weight } \\
(\mathrm{g})\end{array}$ & $\begin{array}{l}\text { cooling rate }\left({ }^{\circ} \mathrm{C} / \mathrm{h}\right) ; \\
\text { start-end }\left({ }^{\circ} \mathrm{C}\right)\end{array}$ & $\begin{array}{l}\text { annealing (centrifuging) } \\
\text { temperature }\left({ }^{\circ} \mathrm{C}\right)\end{array}$ \\
\hline \multirow[t]{4}{*}{$\mathrm{Gd}-\mathrm{Cd}$} & $\mathrm{Gd}_{5} \mathrm{Cd}_{100}$ & LMPF & QC & 2 & 0 & $340, \cdots, 380$ \\
\hline & $\mathrm{Gd}_{1} \mathrm{Cd}_{100}$ & HMC & QC & 6 & $1 ; 455-355$ & 355 \\
\hline & $\mathrm{Gd}_{5} \mathrm{Cd}_{100}$ & LMPF & $1 / 1 \mathrm{AC}$ & 2 & 0 & 400,470 \\
\hline & $\mathrm{Gd}_{5.25} \mathrm{Cd}_{100}$ & $\mathrm{HMC}$ & $1 / 1 \mathrm{AC}$ & 6 & $2 ; 650-500$ & 500 \\
\hline \multirow[t]{4}{*}{$\mathrm{Y}-\mathrm{Cd}$} & $\mathrm{Y}_{5} \mathrm{Cd}_{100}$ & LMPF & QC & 2 & 0 & 340,350 \\
\hline & $\mathrm{Y}_{1} \mathrm{Cd}_{100}$ & HMC & QC & 6 & $1 ; 455-355$ & 355 \\
\hline & $\mathrm{Y}_{5} \mathrm{Cd}_{100}$ & LMPF & $1 / 1 \mathrm{AC}$ & 2 & 0 & $360, \cdots, 400$ \\
\hline & $\mathrm{Y}_{5.25} \mathrm{Cd}_{100}$ & HMC & $1 / 1 \mathrm{AC}$ & 6 & $1 ; 650-500$ & 500 \\
\hline \multirow[t]{4}{*}{$\mathrm{Gd}-\mathrm{Y}-\mathrm{Cd}$} & $\mathrm{Gd}_{2.5} \mathrm{Y}_{2.5} \mathrm{Cd}_{100}$ & LMPF & QC & 2 & 0 & $340, \cdots, 360$ \\
\hline & $\mathrm{Gd}_{0.5} \mathrm{Y}_{0.5} \mathrm{Cd}_{100}$ & HMC & QC & 6 & $1 ; 450-350$ & 350 \\
\hline & $\mathrm{Gd}_{2.5} \mathrm{Y}_{2.5} \mathrm{Cd}_{100}$ & LMPF & $1 / 1 \mathrm{AC}$ & 2 & 0 & $380, \cdots, 400$ \\
\hline & $\mathrm{Gd}_{2.6} \mathrm{Y}_{2.6} \mathrm{Cd}_{100}$ & HMC & $1 / 1 \mathrm{AC}$ & 6 & $1 ; 650-500$ & 500 \\
\hline \multirow{7}{*}{$\underset{\mathrm{Cd}}{\mathrm{Gd}-\mathrm{Zn}-}$} & $\mathrm{Gd}_{5} \mathrm{Zn}_{5} \mathrm{Cd}_{95}$ & LMPF & QC & 2 & 0 & $380, \cdots, 420$ \\
\hline & $\mathrm{Gd}_{1} \mathrm{Zn}_{5} \mathrm{Cd}_{95}$ & HMC & QC & 25 & $0.2 ; 410-350$ & 350 \\
\hline & $\mathrm{Gd}_{5} \mathrm{Zn}_{5} \mathrm{Cd}_{95}$ & LMPF & $1 / 1 \mathrm{AC}$ & 2 & 0 & $450, \cdots, 470$ \\
\hline & $\mathrm{Gd}_{2} \mathrm{Zn}_{5} \mathrm{Cd}_{95}$ & HMC & $1 / 1 \mathrm{AC}$ & 2 & $1 ; 550-450$ & 450 \\
\hline & $\mathrm{Gd}_{5} \mathrm{Zn}_{10} \mathrm{Cd}_{90}$ & LMPF & QC & 2 & 0 & 340,430 \\
\hline & $\mathrm{Gd}_{1.25} \mathrm{Zn}_{10} \mathrm{Cd}_{90}$ & HMC & QC & 2 & $1 ; 450-350$ & 350 \\
\hline & $\mathrm{Gd}_{5} \mathrm{Zn}_{10} \mathrm{Cd}_{90}$ & LMPF & $1 / 1 \mathrm{AC}$ & 2 & 0 & $450, \cdots, 470$ \\
\hline \multirow{4}{*}{$\underset{\mathrm{Cd}}{\mathrm{Gd}-\mathrm{Mg}-}$} & $\mathrm{Gd}_{5} \mathrm{Mg}_{30} \mathrm{Cd}_{70}$ & LMPF & $\mathrm{QC}$ & 2 & 0 & $400, \cdots, 500$ \\
\hline & $\mathrm{Gd}_{1.5} \mathrm{Mg}_{30} \mathrm{Cd}_{70}$ & HMC & $\mathrm{QC}$ & 6 & $1 ; 500-400$ & 500 \\
\hline & $\mathrm{Gd}_{5} \mathrm{Mg}_{30} \mathrm{Cd}_{70}$ & LMPF & $1 / 1 \mathrm{AC}$ & 2 & 0 & 520 \\
\hline & $\mathrm{Gd}_{5} \mathrm{Mg}_{30} \mathrm{Cd}_{70}$ & HMC & $1 / 1 \mathrm{AC}$ & 6 & $2 ; 650-550$ & 550 \\
\hline
\end{tabular}

${ }^{a}$ All chemical compositions are in approximate atom number. Typically, LMPF samples were isothermally annealed for $100 \mathrm{~h}$ at selected temperatures between 340 to $520{ }^{\circ} \mathrm{C}$ until peritectic temperatures were satisfactorily bracketed.

peritectic formation of QCs (which involves dissolution of AC phase) is a slow process at the rather low temperatures where the QCs are actually stable $\left(<\sim 500{ }^{\circ} \mathrm{C}\right) .{ }^{11,13}$

Here, we (re)investigate the $\mathrm{Gd}-\mathrm{Cd}$ system and introduce a procedure, in the following referred to as "low Melt Peritectic Formation (LMPF)", which yields equilibrium samples of QC and liquid at low temperature (down to $330{ }^{\circ} \mathrm{C}$ ) and at conveniently short periods of time (i.e., hours). The procedure allows insight into the peritectic formation of QCs and can be employed for the synthesis of gram-sized batches of QC sample. It further allows the study of thermodynamic relations, including unambiguous bracketing of the peritectic decomposition temperature of QCs. We extend to ternary variants for which $\mathrm{Cd}$ was partially substituted by isovalent $\mathrm{Zn}$ and $\mathrm{Mg}$, or $\mathrm{Gd}$ substituted by Y, and show that substitution decisively alters the decompositions temperature (and hence thermodynamic stability) for QCs, whereas the one for AC phases appears to be very little affected.

\section{EXPERIMENTAL SECTION}

Synthesis. Starting chemicals were commercially obtained as elemental granules from Chempur: Gd and Y with purity 99.99 and 
99.9 at. \% respectively; Alfa Aldrich: $\mathrm{Cd}, \mathrm{Mg}$, and $\mathrm{Zn}$ with purity 99.999 at. \%. Alumina $\left(\mathrm{Al}_{2} \mathrm{O}_{3}\right)$ crucibles, in the form of Canfield Crucible Sets (CCS) were obtained from LSP Industrial Ceramics company. The CCS consists of two flat-bottom cylindrical crucibles and an alumina frit-disc with holes of $\sim 0.7$ to $1 \mathrm{~mm}$ in diameter designed to separate the grains from the melt. ${ }^{14}$ For DSC experiments, custom-made cylindrical stainless steel capsules with inner diameter $\sim 5.1 \mathrm{~mm}$ and height 12.5 mm were employed.

Numerous reactions with the conditions shown in Table 1 were carried out. For each synthesis, the reactants were carefully weighed and sealed inside a glovebox $\left(\mathrm{Ar}\right.$ atmosphere, $<0.1 \mathrm{ppm}$ of $\left.\mathrm{O}_{2}\right)$. All reactions were carried out in CCS and were encapsulated inside stainless steel ampules under inert Ar atmosphere. A commercial multistep programmable muffle furnace was employed for setting the temperatures for the actual reactions. Additionally, an external thermocouple was situated close to the sample position to monitor any deviations between readings of the furnace controller and actual temperature at the sample position; an average of $5{ }^{\circ} \mathrm{C}$ deviations is estimated. Typically, ampules were first heated to $800{ }^{\circ} \mathrm{C}$ during $5 \mathrm{~h}$ and kept for $10 \mathrm{~h}$ to ensure homogeneous melts. When applying HMC, the temperature was slowly lowered (see Table 1) to allow sufficient time for crystal growth during crossing the liquidus line of the binary (pseudobinary) system. When applying LMPF, samples were directly quenched in liquid $\mathrm{N}_{2}$ $\left(\sim-200{ }^{\circ} \mathrm{C}\right)$ and subsequently placed in a preheated furnace in which they were annealed at various temperatures (see Table 1). Both procedures were terminated by isothermally centrifuging off excess melt.

Characterization. The samples were studied with powder X-ray diffraction (PXRD), differential scanning calorimetry (DSC), scanning electron microscopy (SEM) coupled with energy dispersive X-ray spectroscopy (EDX) and electron backscattered detector (EBS), and selected area electron diffraction (SAED) techniques. A Bruker D8 powder diffractometer with $\theta-2 \theta$ diffraction geometry and a $\mathrm{Cu} \mathrm{K}_{\alpha}$ radiation $\left(\mathrm{K}_{\alpha 1}=1.540598 \AA\right.$ and $\left.\mathrm{K}_{\alpha 2}=1.544390 \AA\right)$ was used for collecting PXRD intensities at room temperature. PXRD data were analyzed with the HighScore Plus 3.0 software from PANalytical. ${ }^{15}$ Powdered samples were applied to a zero-diffraction plate, and diffraction patterns were measured in a $2 \theta$ range of $5-90^{\circ}$. In most PXRD experiments, silicon powder was added as an internal standard. DSC measurements were performed with a NETZSCH STA 449 F1 Jupiter instrument. Reactants with total mass $300 \mathrm{mg}$ and nominal compositions $\operatorname{Gd}_{x}(\text { Flux })_{100}(x=0,0.5,1,1.5,2,3,4,5$, 7, and 9; Flux $=$ $\mathrm{Zn}_{5} \mathrm{Cd}_{95}, \mathrm{Zn}_{10} \mathrm{Cd}_{90}$, and $\left.\mathrm{Mg}_{20} \mathrm{Cd}_{80}\right)$ were used. Samples were placed in custom-made stainless steel capsules and sealed under Ar atmosphere by welding; the capsules were preheated to $800{ }^{\circ} \mathrm{C}$ in a muffle furnace prior to DSC measurements. Three heating/cooling cycles to $850{ }^{\circ} \mathrm{C}$ were performed at rates of 25,5 , and $10^{\circ} \mathrm{C} / \mathrm{min}$ under an $\mathrm{Ar}$ flow of $\sim 40 \mathrm{~mL} / \mathrm{min}$. When analyzing isolated QCs and ACs samples, $20-100$ $\mathrm{mg}$ specimen were used at a rate of $10^{\circ} \mathrm{C} / \mathrm{min}$. In all measurements, an empty capsule served as a reference. In some cases, samples were recovered after the measurement and analyzed by PXRD. Scanning electron microscopy (SEM) investigations employed a Zeiss-Merlin instrument equipped with detectors for secondary electron (SE2), energy-selective backscatter electrons (EsB), and energy dispersive Xray (EDX) spectroscopy for elemental analysis with X-Max $80 \mathrm{~mm}^{2}$ Silicon Drift Detector with high sensitivity and at high count rates. Prior to the SEM/EDX experiments, samples were cross-section-polished using $\mathrm{Ar}^{+}$-ion beam in a Cross-Section Polisher SM-09011 instrument from JEOL. Backscattered electron images were recorded with an acceleration voltage of $3 \mathrm{kV}$ and high beam current; EDX data was collected with an acceleration voltage of $20 \mathrm{kV}$ over larger areas $(\sim 100$ $\times 100 \mu \mathrm{m}^{2}$ ) on at least 20 points for each sample. SAED was performed using a JEOL JEM-2100 instrument operating at $200 \mathrm{kV}$ accelerating voltage with double-tilt holder. A small condenser aperture $(70 \mu \mathrm{m})$ and spot size 1 were used.

\section{RESULTS AND DISCUSSION}

Re-Examination of i-GdCd Synthesis and i-GdCd and GdCd6 (AC) Phase Relation. The relevant part of the $\mathrm{Gd}-\mathrm{Cd}$ phase diagram $(0-16$ at. \% Gd) is shown in Figure 2. It is based on the Goldman et al. ${ }^{11}$ study and adapted to include our

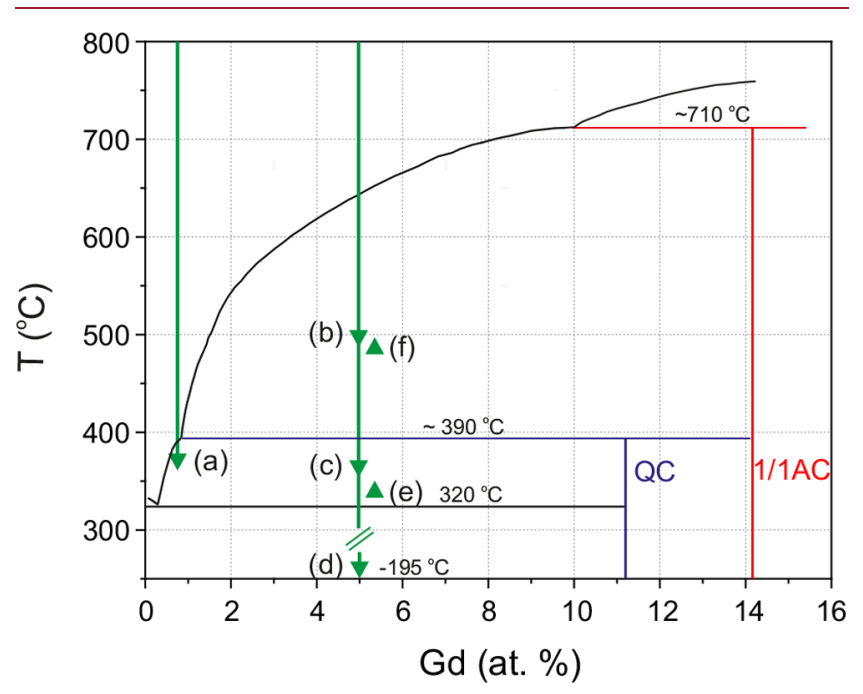

Figure 2. Modified partial phase diagram of the $\mathrm{Gd}-\mathrm{Cd}$ system, adapted from Goldman et al. ${ }^{11}$ Synthesis pathways used in this work are indicated with green arrows/arrowheads: (a) HMC of QC phase, (b) $\mathrm{HMC}$ of AC phase, (c) rapidly cooled (from $800{ }^{\circ} \mathrm{C}$ to $\sim 400{ }^{\circ} \mathrm{C} / \mathrm{h}$ ) mixture $\mathrm{Gd}_{5} \mathrm{Cd}_{100}$, (d) instantly quenched (from $800{ }^{\circ} \mathrm{C}$ to $\sim-200{ }^{\circ} \mathrm{C}$ ) mixture $\mathrm{Gd}_{5} \mathrm{Cd}_{100}$, (e) LMPF of QC (isothermal annealing of product (d) at temperatures between $320^{\circ} \mathrm{C}$ to $\sim 390^{\circ} \mathrm{C}$ ), and (f) LMPF of AC (isothermal annealing of product (d) at temperatures above $\sim 390^{\circ} \mathrm{C}$ ).

findings. The existence of the most Cd-rich phase, $\mathrm{GdCd}_{\sim 8}$, corresponding to the $\mathrm{QC} \mathrm{i}-\mathrm{GdCd}$, was discovered rather independently by Goldman et al. ${ }^{11}$ and Reichmann and Ipser ${ }^{13}$ and reported in 2013 and 2014, respectively. The latter authors obtained i-GdCd by equilibrating elemental mixtures with 2,4 , and 8 at. $\% \mathrm{Gd}$ at $300{ }^{\circ} \mathrm{C}$. The preparation of equilibrium samples within the two-phase field $\mathrm{Cd}+\mathrm{i}-\mathrm{GdCd}$, and also that of a stoichiometric sample ( 11.3 at. \% Gd) at 350 ${ }^{\circ} \mathrm{C}$, required several months. Goldman et al.'s i-GdCd synthesis was based on solution growth by identifying the extremely narrow exposed liquidus line in the range $0.6-0.8$ at. \% Gd, then slowly (over the course 2-3 days) cooling melts to $335^{\circ} \mathrm{C}$ and decanting (or centrifuging) off the remaining, over $99 \%$ Cd-rich, solution. This synthesis pathway ("high melt crystallization", HMC) is indicated in Figure 2 as "(a)" and we could confirm that it results in phase-pure, mm-sized, and well-faceted i-GdCd grains, which are typically covered and glued together by small amounts of residual Cd flux (Figures $3 \mathrm{a}$ and $4 \mathrm{a}$ ).

The exposed liquidus line for the AC phase $\left(\mathrm{GdCd}_{\sim 6}\right)$ stretches over a much broader composition range, from 1.5 to beyond 8 at. \% Gd. In Figure 2, we highlight a scenario corresponding to $\sim 5$ at. \% Gd. Solution growth for $\mathrm{GdCd}_{\sim 6}$ according to $\mathrm{HMC}$ can be performed in a wide temperature region, from about $600{ }^{\circ} \mathrm{C}$ down to the peritectic decomposition line of the QC phase (Figures $3 \mathrm{~b}$ and $4 \mathrm{~b}$ ). As a matter of fact, cooling below this line-even as rapidly as possible (e.g., from 800 to $370{ }^{\circ} \mathrm{C}$ within an hour, which is the fastest cooling rate that we could achieve with our furnace)-and immediate centrifugation will produce mm-sized well-shaped crystals of AC phase (see Figure S1d, Supporting Information, and Figure 4c). Admixed to these AC crystals obtained by rapid cooling is a small fraction of $10-100-\mu \mathrm{m}$-sized pentagon-dodecahedronshaped QC grains. These grains cannot have formed from the 
(a)

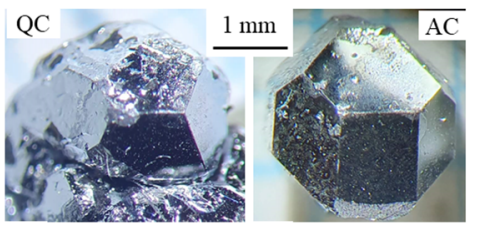

(e)
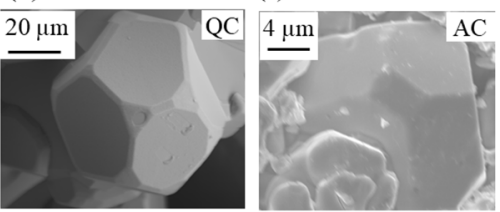

(c)

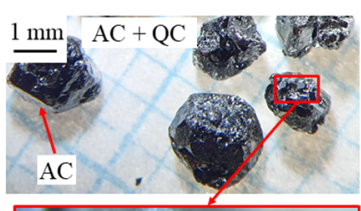

(f)

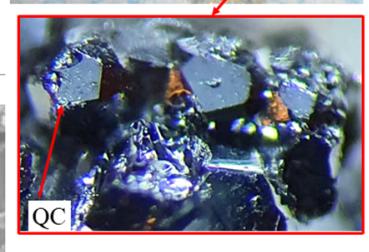

(d)

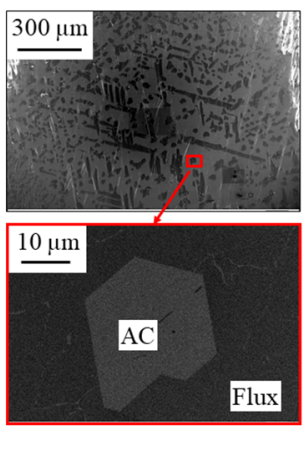

Figure 3. Optical and scanning electron microscopy (SEM) images of products obtained from the different synthesis pathways indicated in Figure 2; hence, the notations (a)-(f) are in accordance with Figure 2. Photographs of (a) HMC QC, (b) HMC AC, and (c) rapidly cooled $\left(\sim 400{ }^{\circ} \mathrm{C} / \mathrm{h}\right) \mathrm{AC} /$ QC phase mixture; SEM images of $(\mathrm{d})$ as-cast $\mathrm{Gd}_{5} \mathrm{Cd}_{100}$ alloy showing several AC grains randomly distributed over Cd-rich matrix (flux); (e) LMPF QC and (f) LMPF AC. Note that similar facets, pentagonal dodecahedron for QCs and rectangular and irregular-hexagonal faces for ACs, were observed on samples obtained from both the HMC (a) and (b) and the LMPF (e) and (f) procedures.

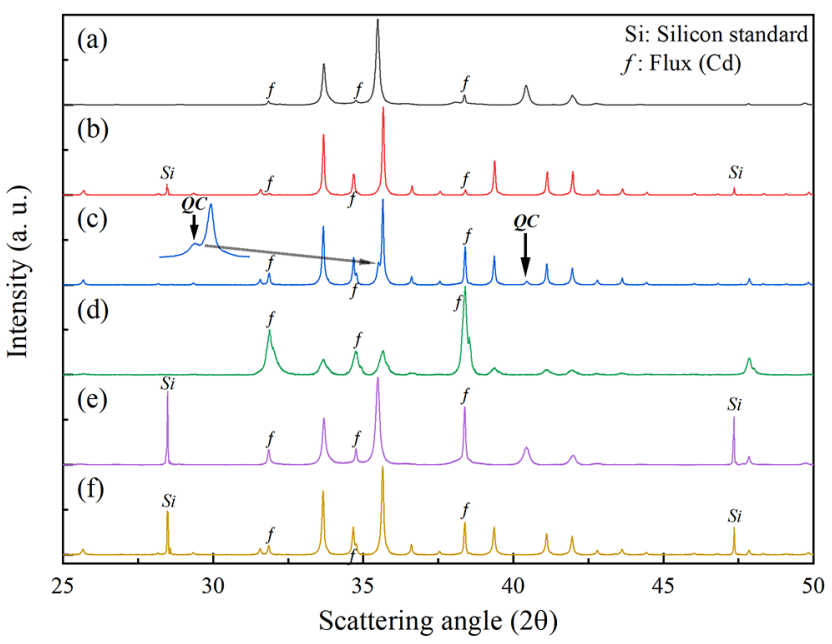

Figure 4. PXRD patterns of products obtained from the different synthesis pathways indicated in Figure 2; hence, the notations (a)-(f) are in accordance with Figure 2. PXRD plots for (a) HMC QC, (b) HMC AC, (c) rapidly furnace-cooled $\left(\sim 400{ }^{\circ} \mathrm{C} / \mathrm{h}\right)$ AC/QC phase mixture, (d) as-cast $\mathrm{Gd}_{5} \mathrm{Cd}_{100}$ alloy, (e) LMPF QC, and (f) LMPF AC. Diffraction background and peaks from $\mathrm{Cu} \mathrm{K}_{\alpha 2}$ radiation has been subtracted from all patterns; Bragg peaks from internal Si-standard $(\mathrm{Si})$ and residual Cd-flux (f) are indicated.

peritectic reaction $\mathrm{AC}+\mathrm{L} \leftrightarrow \mathrm{i}-\mathrm{GdCd}$ but precipitated from the melt to increase the fraction of solid according to the shifted liquidus. If instead of immediate centrifugation the sample is annealed for 4 days, QC grains in addition covered the surface of AC crystals; see Figure 3c. This may indicate a peritectic formation, but AC surfaces could also simply act as nucleation centers for QC precipitation. From PXRD analysis, it could not be deduced whether annealed samples contained a larger fraction of QC phase compared to rapidly cooled ones (cf., Figure $4 \mathrm{c}$ and Figure S2, Supporting Information). We concluded that upon cooling both AC and QC phase nucleate and grow rapidly from the melt but the peritectic reaction $\mathrm{AC}+$ $\mathrm{L} \leftrightarrow \mathrm{i}-\mathrm{GdCd}$ is a rather slow process. A reason for this may be the large crystal size of the AC phase when entering the i-GdCd stability field upon cooling.

As a next step, we rapidly quenched equilibrated melts with $\sim 5$ at. $\% \mathrm{Gd}$ from $800^{\circ} \mathrm{C}$ to liquid- $\mathrm{N}_{2}$ temperature $\left(\sim-200^{\circ} \mathrm{C}\right)$.
This produced samples with $10-20-\mu \mathrm{m}$-sized crystallites of AC-with a streak-like precipitation pattern-embedded in Cdrich matrix (Figures $3 \mathrm{~d}$ and $4 \mathrm{~d}$ ). Heating of such as-cast samples in a DSC apparatus did not show a thermal event associated with (exothermic) peritectic reaction. However, the rapidly quenched samples equilibrated readily in subsequent annealing experiments in a preheated furnace over the course of hours ( 3 to $6 \mathrm{~h}$; see Figure S3 in Supporting Information) at temperatures as low as $340{ }^{\circ} \mathrm{C}$ (Figure $4 \mathrm{e}, \mathrm{f}$ ). As a matter of fact, these equilibrations allowed for unambiguously bracketing the temperature of the peritectic formation of i-GdCd at $390 \pm 5$ ${ }^{\circ} \mathrm{C}$. (For these experiments, we applied a prolonged annealing/ equilibration time of 4 days.) QC samples were afforded as grains with a wide size distribution, ranging from 1 to $100 \mu \mathrm{m}$. The large ones, with sizes $>20 \mu \mathrm{m}$, show the typical pentagonal dodecahedral morphology (Figure 3e). AC samples, which were obtained at temperatures above $400{ }^{\circ} \mathrm{C}$, displayed a similar size distribution. Again, the typical morphology of cubic $1 / 1 \mathrm{AC}$ is easily recognizable for crystallites with $>20 \mu \mathrm{m}$ size (Figure $3 \mathrm{f}$ ). Hence, we infer that the formation of $\mathrm{QC}$ phase from peritectic reactions $\mathrm{AC}+$ liquid ("low melt peritectic formation" $\mathrm{LMPF}$ ) requires $\mathrm{AC}$ phase with a sufficiently small crystallite size $(<\sim 10$ $\mu \mathrm{m}$ ) for overcoming slow kinetics. If this is provided, as, e.g., through rapid quenching, then LMPF is a valuable tool for mapping peritectic reactions and thermodynamic phase relations.

The sluggish formation of $\mathrm{i}-\mathrm{GdCd}$ from peritectic reactions $\mathrm{AC}+$ liquid may also indicate a sluggish decomposition and, accordingly, a certain kinetic stability of i-GdCd. Goldman et al. reported a DSC trace from single grains of $\mathrm{i}-\mathrm{GdCd}$, which corresponded to a broad endothermic signal at around $450{ }^{\circ} \mathrm{C}$. This was interpreted as peritectic decomposition, and accordingly, the stability line for i-GdCd was given as $\sim 450$ ${ }^{\circ} \mathrm{C}$ (cf., Figure 1). Note that this temperature is considerably higher than the one determined from the LMPF experiments. Figure 5a shows the DSC trace of single grains of i-GdCd $(\mathrm{Cd}$ free) as obtained from HMC synthesis (i.e., pathway (a)). Upon heating, there is a weak endothermic signal at around $540^{\circ} \mathrm{C}$. The strong signal with onset at about $700{ }^{\circ} \mathrm{C}$ has to correspond to decomposition of AC phase and, successively, complete melting. These two events are resolved upon cooling. The cooling trace indicates that the liquidus temperature for the $\mathrm{i}$ GdCd composition (11.3 at. \% Gd; see Table 2) is around 730 


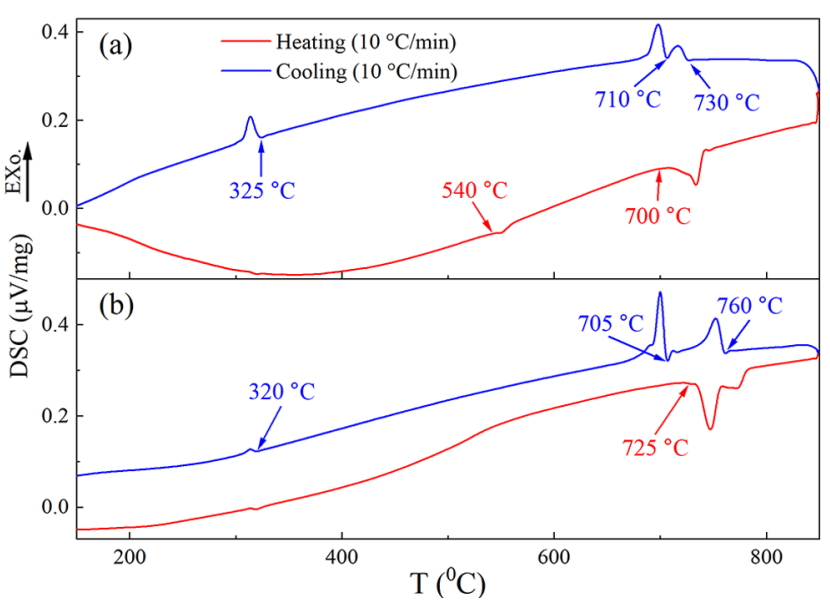

Figure 5. DSC traces of QC (a) and AC phase (b) obtained from HMC. Temperatures of endothermic and exothermic events are indicated. The exothermic peaks close to 325 and $320^{\circ} \mathrm{C}$ correspond to solidification of Cd-rich melt.

${ }^{\circ} \mathrm{C}$, and that the peritectic formation of AC phase occurs near $710{ }^{\circ} \mathrm{C}$. Note that both temperatures are lower than in the Goldman et al. phase diagram, ${ }^{11}$ but in good agreement with that of Reichmann and Ipser. ${ }^{13}$ We attribute the signal at $540{ }^{\circ} \mathrm{C}$ to the (kinetically delayed) peritectic decomposition of $\mathrm{i}-\mathrm{GdCd}$ (which occurred in the Goldman et al. experiment at $\sim 450^{\circ} \mathrm{C}$ ). To support this, experiments were performed on QC specimens, which simulated the heating rate of the DSC experiment in a regular furnace and were terminated by liquid- $\mathrm{N}_{2}$ quenching. These experiments showed survival of the QC phase up to at least $520{ }^{\circ} \mathrm{C}$ (see Figure S4 in Supporting Information files). The DSC trace of the corresponding AC sample does not show a signal below decomposition and melting, for which the onset is at about $725{ }^{\circ} \mathrm{C}$ (Figure 5b). On cooling, the event at $760{ }^{\circ} \mathrm{C}$ corresponds to the liquidus for the $\mathrm{AC}$ composition (14.2 at. \% $\mathrm{Gd}$; see Table 2) and the one at $705{ }^{\circ} \mathrm{C}$ to peritectic $\mathrm{AC}$ formation, which should be at the same temperature as for the QC composition (cf., Figure 5a). For plotting the phase diagram shown in Figure 2, we used liquidus and peritectic temperatures as obtained in our work.

Chemical Modification of Gd-Cd-Pseudobinary Gd$(\mathbf{C d}, \mathbf{Z n})$ and $\mathbf{G d}(\mathbf{C d}, \mathbf{M g})$. We then embarked on the investigation of the pseudobinary $\mathrm{Gd}-(\mathrm{Cd}, \mathrm{Zn})$ and $\mathrm{Gd}$ $(\mathrm{Cd}, \mathrm{Mg})$ phase diagrams, with the aim to determine the limiting compositions of $\mathrm{i}-\mathrm{QC}$ and $\mathrm{AC}$ phases upon replacing $\mathrm{Cd}$ for isovalent $\mathrm{Zn}$ and $\mathrm{Mg}$, and to establish the changes of the peritectic decomposition temperatures for the pseudobinary phases. The liquidus line was conveniently mapped with DSC analysis, and the result is exemplified with $\mathrm{Gd}-\mathrm{Cd}_{95} \mathrm{Zn}_{5}$ (Figure 6). With respect to binary $\mathrm{Gd}-\mathrm{Cd}$, we observed a flattening of the exposed liquidus line for the $\mathrm{i}-\mathrm{QC}$ phase, in conjunction with a shift of the eutectic point from $0.3 \% \mathrm{Gd}$ to between $0.5 \%$ and $1 \% \mathrm{Gd}$. This allowed the application of a wider compositiontemperature window for high melt crystallization/solution growth of i-QC phase for the $\mathrm{Gd}-\left(\mathrm{Cd}_{95} \mathrm{Zn}_{5}\right)$ system. As a consequence, pentagonal dodecahedral-shaped grains of 4-5 $\mathrm{mm}$ size could be conveniently obtained (cf., inset in Figure 6).

For studying the peritectic formation of i-Gd- $\left(\mathrm{Cd}_{95} \mathrm{Zn}_{5}\right)$, we again employed $\sim 5$ at. \% Gd mixtures (see Figure S1b and Table $S 1$ in the Supporting Information for further information). The peritectic formation reaction was bracketed at $425 \pm 5^{\circ} \mathrm{C}$, which is higher than that for binary i-GdCd. In contrast, the peritectic formation/decomposition of the corresponding $\mathrm{AC}$ phase is decreased by about $5^{\circ} \mathrm{C}$ (from 710 to $705^{\circ} \mathrm{C}$ ) and thus changed only little. The ratio of $\mathrm{Cd}$ and $\mathrm{Zn}$ in both $\mathrm{QC}$ and $\mathrm{AC}$ samples was close to the nominal composition. By increasing the $\mathrm{Zn}$ content further, it was possible to maintain pseudobinary conditions for up to $\mathrm{Cd}_{88} \mathrm{Zn}_{12}$. At higher $\mathrm{Zn}$ concentrations, interference with a $\mathrm{Zn}$-rich phase, $\mathrm{Gd}_{2} \mathrm{Zn}_{14.6} \mathrm{Cd}_{2.4}$ with the hexagonal $\mathrm{Th}_{2} \mathrm{Ni}_{17}$ structure, was observed. This indicated phase separation into $\mathrm{Cd}$ - and $\mathrm{Zn}$-rich compounds. Higher $\mathrm{Zn}$ concentrations, beyond $\mathrm{Cd}_{85} \mathrm{Zn}_{15}$, were not investigated. The boundary composition of pseudobinary Gd- $(\mathrm{Cd}, \mathrm{Zn}) \mathrm{QC}$ and $\mathrm{AC}$ is $\mathrm{Cd}_{88} \mathrm{Zn}_{12}$ (see Table 2 for compositional analysis results of $\mathrm{Gd}-(\mathrm{Cd}, \mathrm{Zn})$ samples $)$.

With respect to $\mathrm{Mg}$ substitution, Labib et al. recently performed an extensive investigation into pseudobinary RE(Cd, Mg) QCs and their cubic AC phases, covering a broad range of $\mathrm{RE}(\mathrm{RE}=\mathrm{Y}, \mathrm{Sm}, \mathrm{Gd}, \mathrm{Tb}, \mathrm{Dy}, \mathrm{Ho}, \mathrm{Er}, \mathrm{Tm}) .^{16}$ Remarkably, these authors observed that alloys constituting $\mathrm{Cd}_{100-x} \mathrm{Mg}_{x}(20<x<30)$ and $12-16$ at. \% RE could be equilibrated-after initial melting at $850{ }^{\circ} \mathrm{C}$-in the temperature interval $400-600^{\circ} \mathrm{C}$, to yield ternary QC and AC phases. Thus, the presence of $\mathrm{Mg}$ appears to promote the kinetics for peritectic QC formation. In addition, as a distinguished feature, pseudobinary $\mathrm{Mg}$ systems revealed the presence of 2/1 AC phases (instead of $1 / 1$ as in the binary RE-Cd systems), apart from $\mathrm{RE}=\mathrm{Sm}, \mathrm{Gd}$. In our work, we again focused on $\mathrm{RE}=\mathrm{Gd}$ and found that the maximum solubility of $\mathrm{Mg}$ in ternary i$\mathrm{Gd}(\mathrm{Cd}, \mathrm{Mg})$ and the $1 / 1 \mathrm{AC}$ phase is close to $20 \%$ (see Table 2). Accordingly, we applied $\mathrm{Cd}_{80} \mathrm{Mg}_{20}$ for studying the pseudobinary Gd-(Cd,Mg) phase diagram (see Figure S1a and Table S1 in the Supporting Information for further information).

Figure 7 displays a summary of pseudobinary phase diagrams, to visualize the changes with respect to binary $\mathrm{Gd}-\mathrm{Cd}$. As already mentioned, with $\mathrm{Zn}$ a flattening of the liquidus line is observed, concomitant with a shift of the eutectic to between 0.5

Table 2. Summary of Experimental Results for QCs and ACs ${ }^{a}$

\begin{tabular}{|c|c|c|c|c|c|c|c|}
\hline \multirow[b]{2}{*}{ system } & \multicolumn{3}{|c|}{ composition (at.\%) from EDX } & \multicolumn{2}{|c|}{$\begin{array}{l}\text { peritectic decomposition } \\
\text { temp. }\left({ }^{\circ} \mathrm{C}\right)\end{array}$} & \multicolumn{2}{|c|}{ lattice parameter $(\AA)$} \\
\hline & nominal (starting) & $\mathrm{QC}$ & $\mathrm{AC}$ & QC & $\mathrm{AC}^{a}$ & $1 / 1 \mathrm{AC}(a)$ & $\mathrm{QC}\left(a_{6 \mathrm{D}}\right)$ \\
\hline 1 & $\mathrm{Gd}_{5} \mathrm{Cd}_{100}$ & Gd11.4(8)Cd88.6(8) & Gd14.0(7)Cd86.0(7) & $390(5)$ & $710(5)$ & $15.514(2)$ & $7.970(2)$ \\
\hline 2 & $\mathrm{Y}_{5} \mathrm{Cd}_{100}$ & Gd11.3(5)Cd88.7(5) & Gd13.2(9)Cd86.8(9) & $350(5)$ & $690(5)$ & $15.481(2)$ & $7.953(2)$ \\
\hline 3 & $\left(\mathrm{Gd}_{2.5} \mathrm{Y}_{2.5}\right) \mathrm{Cd}_{100}$ & Gd5.9(5)Y5.2(6)Cd88.9(9) & Gd7.5(4)Y6.8(5)Cd85.7(9) & $370(5)$ & $695(5)$ & $15.501(3)$ & $7.963(3)$ \\
\hline 4 & $\mathrm{Gd}_{5}\left(\mathrm{Cd}_{95} \mathrm{Zn}_{5}\right)$ & Gd11(1)Cd83(1)Zn6(1) & Gd13.8(5)Cd80.0(7)Zn6.2(4) & $425(5)$ & $705(5)$ & $15.426(1)$ & $7.925(1)$ \\
\hline 5 & $\mathrm{Gd}_{5}\left(\mathrm{Cd}_{90} \mathrm{Zn}_{10}\right)$ & Gd11(1)Cd79(2)Zn11(1) & Gd13.3(3)Cd74.7(5)Zn12.0(4) & $440(5)$ & $700(5)$ & $15.389(2)$ & $7.906(2)$ \\
\hline 6 & $\mathrm{Gd}_{5}\left(\mathrm{Cd}_{70} \mathrm{Mg}_{30}\right)$ & $\operatorname{Gd} 11(1) \mathrm{Cd} 70(3) \operatorname{Mg} 19(3)$ & $\operatorname{Gd} 14(1) \operatorname{Cd} 67(1) \operatorname{Mg} 19(1)$ & $520(5)$ & $670(5)$ & $15.534(1)$ & $7.980(9)$ \\
\hline
\end{tabular}

${ }^{a}$ Obtained from DSC experiments. 


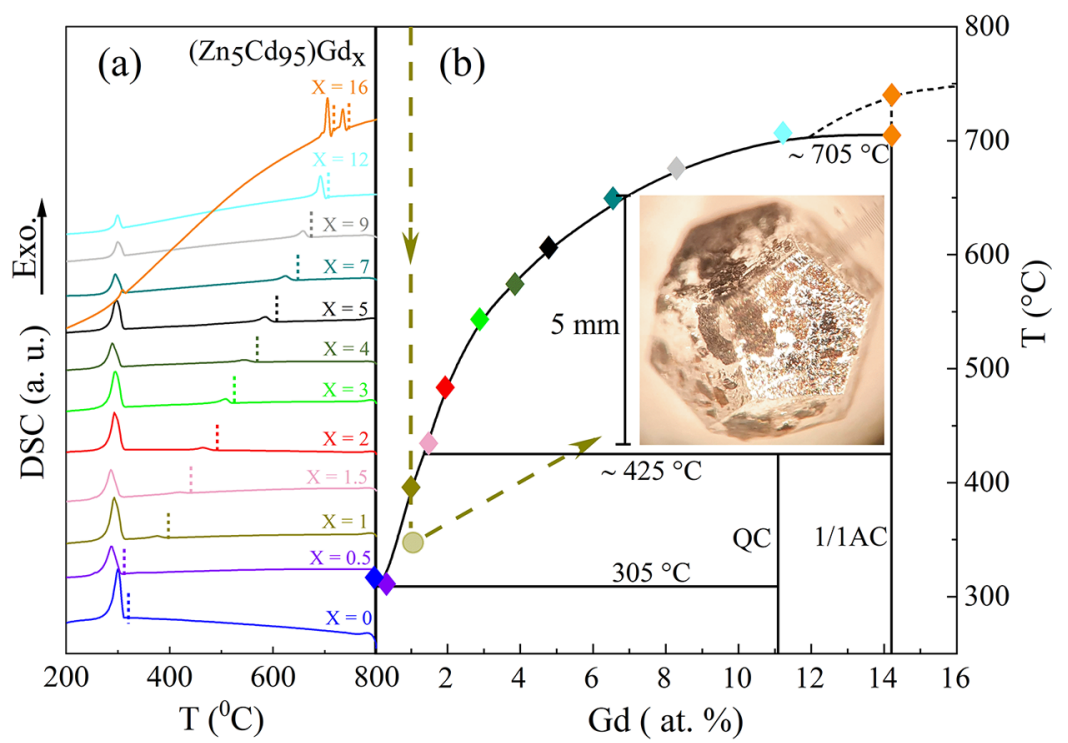

Figure 6. Thermal analysis and partial-pseudobinary phase diagram for the Gd- $\left(\mathrm{Zn}_{5} \mathrm{Cd}_{95}\right)$ pseudobinary system. (a) DSC cooling curves for reaction mixtures $\left(\mathrm{Zn}_{5} \mathrm{Cd}_{95}\right) \mathrm{Gd}_{x}$, where $x$ ranges from 0 to 16; the specific temperatures used to map the liquidus line in (b) are indicated by broken lines in each plot. (b) Sketch of the partial pseudobinary phase diagram. The decomposition temperature of the QC phase $\left(\sim 425^{\circ} \mathrm{C}\right)$ was estimated from LMPF experiments. The inset photographic image is an isolated QC grain obtained from HMC using a nominal starting composition $\mathrm{Zn}_{5} \mathrm{Cd} \mathrm{d}_{95} \mathrm{Gd}_{1}$.

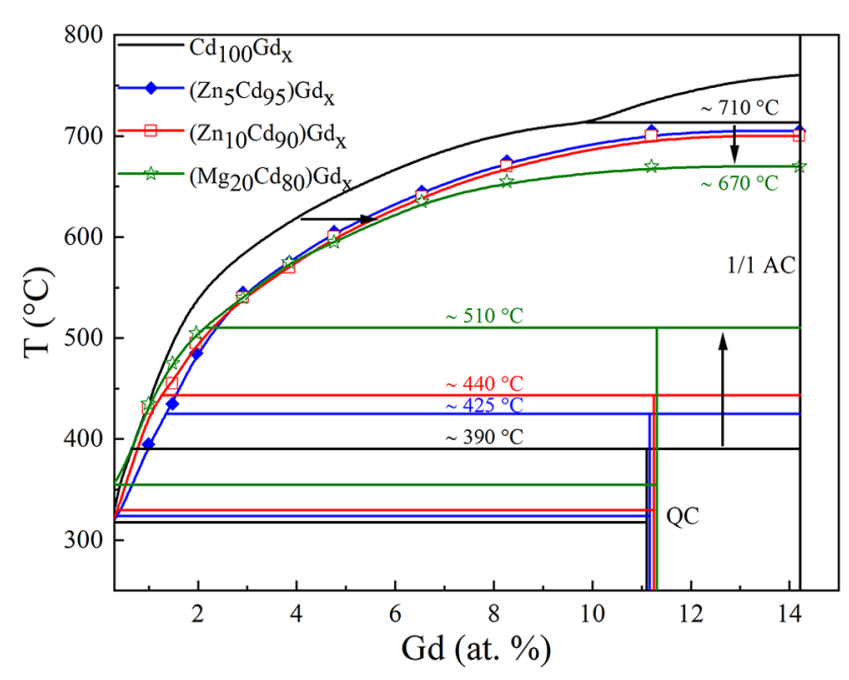

Figure 7. Combined plots of phase diagrams for binary $\mathrm{Gd}-\mathrm{Cd}$ and pseudobinary $\mathrm{Gd}-\left(\mathrm{Zn}_{5} \mathrm{Cd} \mathrm{d}_{95}\right)$, Gd- $\left(\mathrm{Zn}_{10} \mathrm{Cd}_{90}\right)$, and $\mathrm{Gd}-\left(\mathrm{Mg}_{20} \mathrm{Cd}_{80}\right)$. The pseudobinary diagrams have been established from cooling curves of DSC experiments (cf., Figure 6a). The decomposition temperatures of the QC and AC phases were obtained from LMPF and DSC experiments, respectively. General trends of changes in the liquidus line, and $\mathrm{QC}$ and $\mathrm{AC}$ peritectic decomposition temperatures, are indicated by arrows.

and 1 at. \% Gd. This is also the case for the $(\mathrm{Cd}, \mathrm{Mg})$ pseudobinary system. The peritectic temperature for the i-QC phases increases with $\mathrm{Zn}$ and especially with $\mathrm{Mg}$ substitution. At the same time, the peritectic temperatures of the AC phases appear to be only slightly affected upon $\mathrm{Zn}$ substitution, whereas $\mathrm{Mg}$ substitution results in a significant decrease. Accordingly, we conjecture that divalent metal substitution leads to significant thermodynamic stabilization of the i-QC phase, whereas the AC phase becomes rather destabilized. Assessing the thermodynamic stability of QCs is still an open question, and we infer that for (pseudo)binary i-QCs their peritectic decomposition temperatures represent at least a qualitative indicator. Yet, one has to be aware that this temperature is also determined by the Gibbs energy curves of the AC phase and the liquid.

Next, we compare the PXRD pattern to analyze overall trends of the substitution (Figure 8). The indices for i-GdCd QC have been obtained by comparing the present pattern with the one reported by Goldman et al. ${ }^{11}$ for the same system; only indices of strong reflections are shown. The atomic/metallic radii of $\mathrm{Cd}$ and $\mathrm{Mg}$ are very similar, $1.52 \AA$ for $\mathrm{Cd}$ and $1.60 \AA$ for $\mathrm{Mg}$. Consequently, the lattice and quasilattice parameters for the $\mathrm{Mg}$-substituted samples are very similar to the binary ones. In contrast, $\mathrm{Zn}$ substitution results in a significant contraction of lattice parameters (the metallic radius of $\mathrm{Zn}$ is $1.37 \AA$ ) which actually exceeds by far the contraction trend observed for RE-Cd when going from $\mathrm{Gd}$ to $\mathrm{Tm}$. The lattice constant $(a)$ of a $1 / 1$ approximant to an $\mathrm{i}-\mathrm{QC}$ is related to the six-dimensional hypercubic lattice constant $\left(a_{6 \mathrm{D}}\right)$ of the $\mathrm{i}-\mathrm{QC}$ via the relation: $a=\sqrt{\frac{2}{2+\tau}}(1+\tau) a_{6 \mathrm{D}}$, where $\tau=(1+\sqrt{5}) / 2$ is the golden mean constant. ${ }^{17,18}$ The calculated $a_{6 \mathrm{D}}$ values of the present compounds are listed in Table 2 together with their corresponding $a$ 's, which were obtained from standard PXRD analysis. In addition, due to the fact that the $\mathrm{Gd}-\mathrm{Cd}-\mathrm{Zn}$ system is an original system presented in this work, we further confirm its quasicrystallinity using selected area electron diffraction (SAED) experiment on the $\mathrm{Gd}_{11} \mathrm{Cd}_{77} \mathrm{Zn}_{12}$ sample. The fivefold, threefold, and twofold diffraction symmetries, which are characteristic features of i-QCs, were observed as shown in Figure S5 in Supporting Information.

Chemical Modification of $\mathrm{Gd}-\mathrm{Cd}-$ Pseudobinary $(G d, Y) C d$. Lastly, we also investigated the effect of RE replacement to the peritectic temperatures of the $\mathrm{i}-\mathrm{QC}$ and AC phases. We chose $\mathrm{Y}$ which has virtually the same size as Gd (the metallic radii is $1.801 \AA$ for Gd and Y) and is also clearly trivalent in intermetallic compounds, but nonmagnetic (i.e., $\mathrm{Y}$ does not possess $\mathrm{f}$ electrons). It appears that $\mathrm{Y}$ and $\mathrm{Gd}$ display a complete solid solution behavior. We report the peritectic temperatures for the QC and AC phases of $\left(\mathrm{Gd}_{50} \mathrm{Y}_{50}\right)-\mathrm{Cd}$ and $\mathrm{Y}_{100}-\mathrm{Cd}$ (see Figure $\mathrm{S} 1 \mathrm{c}$ and Table $\mathrm{S} 1$ in the Supporting 


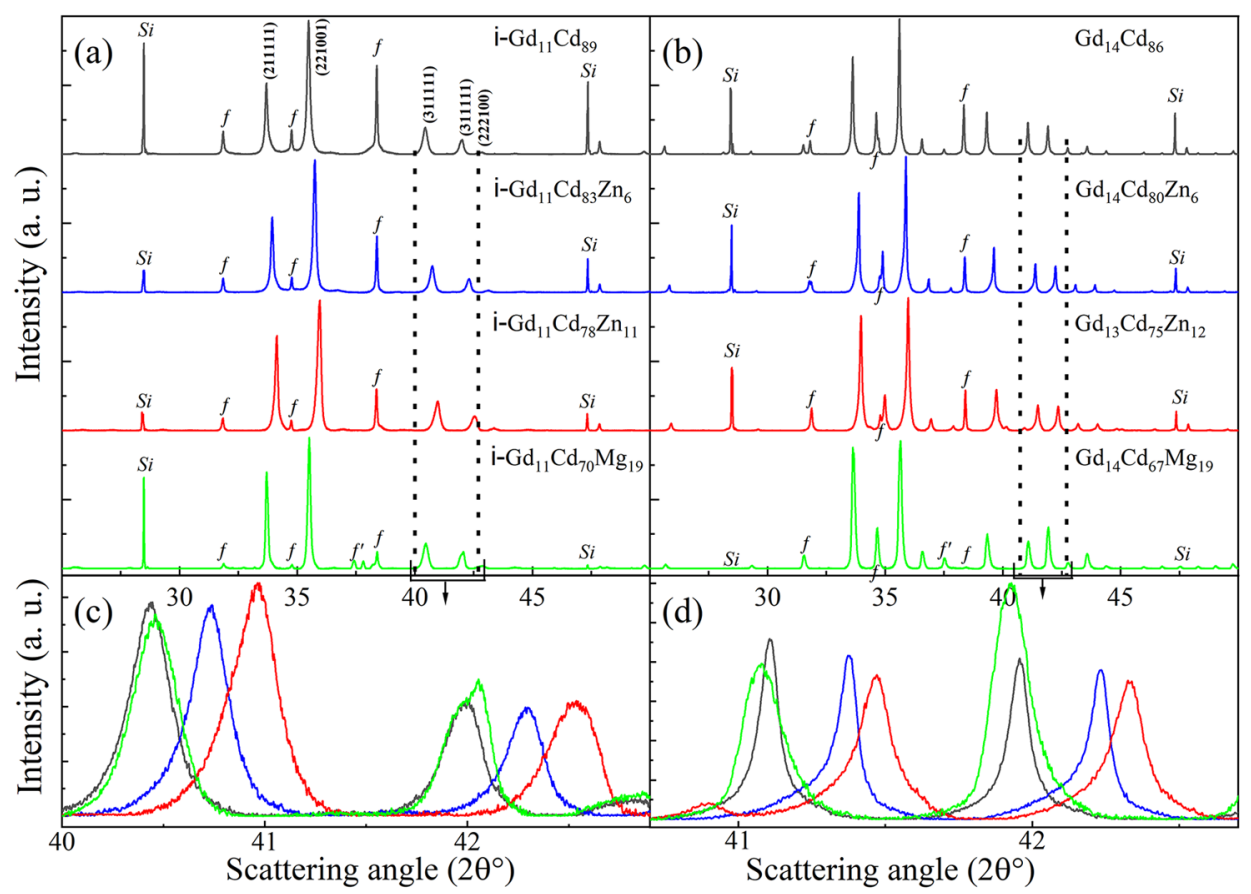

Figure 8. PXRD patterns of (a) QCs and (b) 1/1 ACs obtained from LMPF. To indicate the systematic shift in diffraction peaks, zoomed in sections of (a) and (b), which are marked by broken lines in each plot, are shown in (c) and (d), respectively. The shifts are attributed to the replacement of Cd by $\mathrm{Mg}$ and $\mathrm{Zn}$. Diffraction background and peaks from $\mathrm{Cu} \mathrm{K} \mathrm{K}_{\alpha 2}$ radiation have been subtracted from each pattern; Bragg peaks from internal Si-standard $(\mathrm{Si})$ and residual flux $\left(f\right.$ and $\left.f^{\prime}\right)$ are indicated.

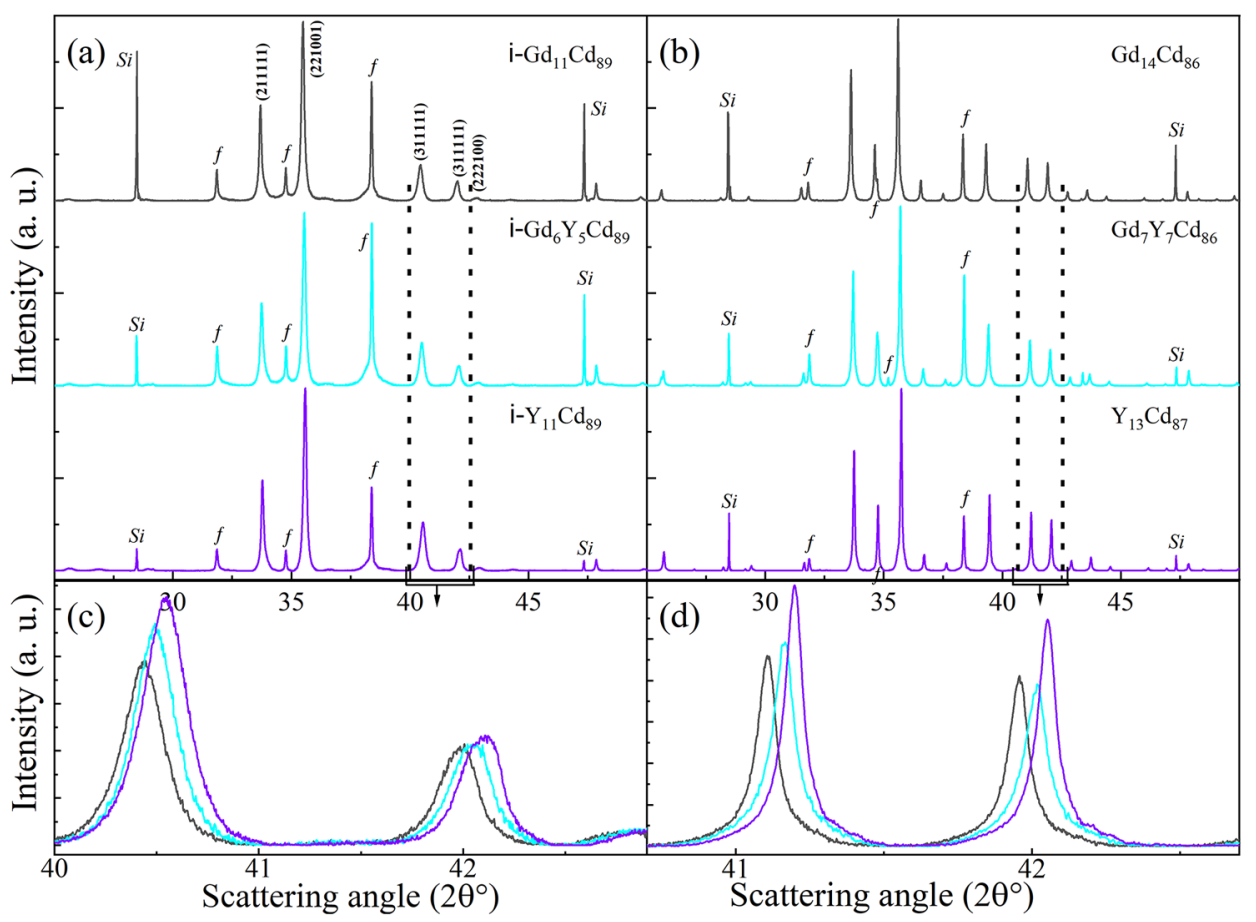

Figure 9. PXRD patterns of (a) QCs and (b) 1/1 ACs obtained from LMPF. To indicate the systematic shift in diffraction peaks, zoomed in sections of (a) and (b), which are marked by broken lines in each plot, are shown in (c) and (d), respectively. The shifts are attributed to the partial and full replacement of $\mathrm{Gd}$ by Y. Diffraction background and peaks from $\mathrm{Cu} \mathrm{K}_{\alpha 2}$ radiation have been subtracted from each pattern; Bragg peaks from internal $\mathrm{Si}$-standard $(\mathrm{Si})$ and residual Cd-flux $(\mathrm{f})$ are indicated.

Information for further information). Surprisingly, with $\mathrm{Y}$ the peritectic temperature (and hence stability) of the QC phase decreases substantially with respect to i-GdCd: it is $370{ }^{\circ} \mathrm{C}$ for i$\left(\mathrm{Gd}_{50} \mathrm{Y}_{50}\right)-\mathrm{Cd}$ and just $350^{\circ} \mathrm{C}$ for i-YCd. In contrast, and similar to the divalent metal substitutions, the peritectic temperature of the corresponding $\mathrm{AC}$ phases is changed only slightly with respect to binary $\mathrm{GdCd}_{\sim 6}$, i.e., it decreases from 710 to $695{ }^{\circ} \mathrm{C}$ for $\left(\mathrm{Gd}_{50} \mathrm{Y}_{50}\right)-\mathrm{Cd}$ to $690{ }^{\circ} \mathrm{C}$ for $\mathrm{YCd}_{\sim 6}$; see Figure $\mathrm{S} 6$ in 
Supporting Information. The PXRD patterns of the ternary (Y,Gd)-Cd phases are shown in Figure 9, in comparison with the binary $\mathrm{Gd}-\mathrm{Cd}$ and $\mathrm{Y}-\mathrm{Cd}$ ones. One notices a slight contraction of both the quasilattice parameter for the $\mathrm{QC}$ and the lattice parameter for the AC phase with Y substitution, in agreement with previous work. ${ }^{11,16,19,20}$

Table 2 gives a summary of compositions, peritectic temperatures, and lattice parameters for the investigated QCs and ACs. As initially mentioned, the stability of QCs with respect to $3 \mathrm{D}$ periodic ACs is still not understood. Here, we argue that the peritectic (decomposition) temperature provides sought-after stability information, yet more investigations are necessary to pin down the atomistic origin of increased/ decreased stability. There has been a long discussion regarding to which degree QCs are energy- or entropy-stabilized. ${ }^{7}$ The role of entropic contributions is a difficult question because of the poorly understood structural disorder and the peculiarity of phason modes. ${ }^{4}$ Yet, one would assume a higher entropy for chemically disordered ternary systems (both QCs and ACs).

The enthalpic stability of QC is typically discussed in terms of the empirical parameters "average number of valence electrons per atom", $(e / a)$, and "atomic size ratio" $R=r_{A} / r_{B}$ where A represents the (larger sized) minority and $\mathrm{B}$ the majority constituent. Here, A is $\mathrm{RE}$ and $\mathrm{B} \mathrm{Cd}$ (or $\mathrm{Cd}-\mathrm{Mg}, \mathrm{Cd}-\mathrm{Zn}$ ). Sometimes the atomic size ratio is also expressed as concentration weighed "effective atomic size ratio" $R=\left(c_{\mathrm{A}} \times\right.$ $\left.r_{\mathrm{A}}\right) /\left(c_{\mathrm{B}} \times r_{\mathrm{B}}\right){ }^{21}$ The $e / a$ criterion relates to the so-called HumeRothery stabilization of QCs according to which the Fermi level is adjusted to a pseudogap in the electronic density of states. The atomic size ration criterion is based on the conditions of effective packing differently sized rigid sphere like atoms $A$ and $B$ forming the dodecahedron (B), icosahedron (A), and icosidodecahedron shells (B) in the Tsai-type cluster building units of QCs (and also ACs). The ideal value for $R$ for achieving the maximum packing volume fraction of spheres is $1.288 .{ }^{22}$ In our case, all systems are isoelectronic, so the $e / a$ criterion does not account for changes in stability. In addition, the size ratio criterion is not very strong, since the exchange of $\mathrm{Gd}$ for $\mathrm{Y}$ or $\mathrm{Cd}$ for $\mathrm{Mg}$ has only minor consequences to $R$, yet the peritectic temperatures of $\mathrm{i}$ YCd and i-Gd $\left(\mathrm{Cd}_{80} \mathrm{Mg}_{20}\right)$ differ by $170{ }^{\circ} \mathrm{C}$.

In order to explain the increased stability i- $\mathrm{Gd}(\mathrm{Cd}, \mathrm{Mg})$ and $\mathrm{i}$ $\mathrm{Gd}(\mathrm{Cd}, \mathrm{Zn})$, we think that the introduction of polarity in the network formed by the B-type atoms plays an important role. The (Allred-Rochow) electronegativity for $\mathrm{Cd}, \mathrm{Zn}$, and $\mathrm{Mg}$ is $1.46,1.66$, and 1.23 , respectively. Thus, substitution of $\mathrm{Cd}$ for $\mathrm{Zn}$ and $\mathrm{Mg}$ will introduce opposite bond polarity between network forming atoms, with $\mathrm{Cd}$ positively polarized with respect to $\mathrm{Zn}$ and negatively polarized with respect to $\mathrm{Mg}$. To elaborate on this, it is necessary to perform a detailed structure analysis of the pseudobinary $\mathrm{i}-\mathrm{QC}$ and $\mathrm{AC}$ phases in order to reveal and analyze the site distribution patterns. In addition, we do not have an explanation for the astonishingly large difference between $\mathrm{i}-\mathrm{YCd}$ and i-GdCd. Why is the QC with Gd bearing a localized magnetic moment considerably more stable? i-GdGd exhibits spin glass behavior, which is not supposed to contribute to structure/phase stability.

\section{CONCLUSIONS}

We reliably determined temperatures for the peritectic reaction $\mathrm{AC}+\mathrm{L} \leftrightarrow \mathrm{i}-\mathrm{QC}$ for $\mathrm{i}-\mathrm{Gd}-\mathrm{Cd}$ and ternary variants where $\mathrm{Cd}$ is partially replaced by isovalent $\mathrm{Zn}$ or $\mathrm{Mg}$, as well as when the $4 \mathrm{f}$ element $\mathrm{Gd}$ is replaced by nonmagnetic Y. We argue that these temperatures reflect the thermodynamic stability of (pseudo)- binary i-QCs and find that $\mathrm{Zn}$ and especially $\mathrm{Mg}$ substitution leads to significant stabilization, whereas $\mathrm{Y}$ substitution leads to destabilization. The peritectic temperatures for i-YCd and i$\mathrm{Gd}(\mathrm{Cd}, \mathrm{Mg})$ differ by $170{ }^{\circ} \mathrm{C}$. As a consequence of the increased stabilizations of $\mathrm{Zn}$ and $\mathrm{Mg}$ substituted QCs, a broader composition/temperature window for accessing the $\mathrm{QC}$ phase via the high-melt-crystallization was achieved, and grains with sizes of several millimeters were grown especially in the Gd$\mathrm{Cd}-\mathrm{Zn}$ system. An alternative syntheses pathway, referred to here as low-melt-peritectic-formation, was employed to prepare high-yield (gram-sized) samples in relatively short times (less than a day) and to determine the peritectic reaction between QC and AC. As a next step, it will be important to identify the atomistic origin of increased/decreased stability.

\section{ASSOCIATED CONTENT}

\section{SI Supporting Information}

The Supporting Information is available free of charge at https://pubs.acs.org/doi/10.1021/acs.cgd.1c00147.

Optical and SEM images of synthesis products, plots of PXRD and DSC results, table for compositional analysis (EDX) results (PDF)

\section{AUTHOR INFORMATION}

\section{Corresponding Author}

Girma Gebresenbut - Department of Chemistry-Ångström Laboratory, Uppsala University, 75121 Uppsala, Sweden;

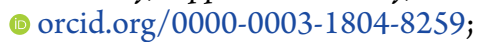

Email: girma.gebresenbut@kemi.uu.se

\section{Authors}

Daniel Eklöf - Department of Materials and Environmental Chemistry, Stockholm University, 10691 Stockholm, Sweden

Alisa Gordeeva - Department of Materials and Environmental Chemistry, Stockholm University, 10691 Stockholm, Sweden

Takayuki Shiino - Department of Materials Science and Engineering, Uppsala University, 75121 Uppsala, Sweden

Ulrich Häussermann - Department of Materials and Environmental Chemistry, Stockholm University, 10691 Stockholm, Sweden; (1) orcid.org/0000-0003-2001-4410

Complete contact information is available at: https://pubs.acs.org/10.1021/acs.cgd.1c00147

\section{Author Contributions}

The manuscript was written through contributions of all authors. All authors have given approval to the final version of the manuscript.

\section{Funding}

We thank the Knut and Alice Wallenberg Foundation (grant number KAW 2018.0019) and the Carl Tryggers Stiftelse for Vetenskaplig Forskning (Grant No. CTS 19:235).

\section{Notes}

The authors declare no competing financial interest.

\section{ABBREVIATIONS}

QCs, quasicrystals; ACs, approximants of quasicrystals; LMPF, low melt peritectic formation; HMC, high melt crystallization; $\mathrm{RE}$, rare earth elements; PXRD, powder X-ray diffraction; SCXRD, single crystal X-ray diffraction; DSC, differential scanning calorimetry; SEM, scanning electron microscopy; EDX, energy dispersive X-ray 


\section{REFERENCES}

(1) Shechtman, D.; Blech, I.; Gratias, D.; Cahn, J. Metallic Phase with Long-Range Orientational Order and No Translational Symmetry. Phys. Rev. Lett. 1984, 53 (20), 1951-1953.

(2) Hafner, J.; Krajčí, M. Electronic structure and stability of quasicrystals: Quasiperiodic dispersion relations and pseudogaps. Phys. Rev. Lett. 1992, 68 (15), 2321-2324.

(3) Tsai, A.-P.; Guo, J.; Abe, E.; Takakura, H.; Sato, T. A stable binary quasicrystal. Nature 2000, 408 (6812), 537-538.

(4) de Boissieu, M. Stability of quasicrystals: energy, entropy and phason modes. Philos. Mag. 2006, 86 (6-8), 1115-1122.

(5) Henley, C.; De Boissieu, M.; Steurer, W. Discussion on clusters, phasons and quasicrystal stabilisation. Philos. Mag. 2006, 86 (6-8), $1131-1151$.

(6) Steurer, W. On a realistic growth mechanism for quasicrystals. $Z$. Anorg. Allg. Chem. 2011, 637 (13), 1943-1947.

(7) Steurer, W. Quasicrystals: What do we know? What do we want to know? What can we know? Acta Crystallogr., Sect. A: Found. Adv. 2018, $74(1), 1-11$.

(8) Turulski, J. Dimension of the Gibbs function topological manifold: 2 . Thermodynamically stable binary quasicrystals: Reality or artefact? J. Math. Chem. 2015, 53 (2), 517-526.

(9) Guo, J.; Abe, E.; Tsai, A. Stable icosahedral quasicrystals in binary $\mathrm{Cd}-\mathrm{Ca}$ and $\mathrm{Cd}-\mathrm{Yb}$ systems. Phys. Rev. B: Condens. Matter Mater. Phys. 2000, 62 (22), R14605.

(10) Canfield, P.; Caudle, M.; Ho, C.-S.; Kreyssig, A.; Nandi, S.; Kim, M.; Lin, X.; Kracher, A.; Dennis, K.; McCallum, R. Solution growth of a binary icosahedral quasicrystal of Sc $12 \mathrm{Zn} \mathrm{88.} \mathrm{Phys.} \mathrm{Rev.} \mathrm{B:} \mathrm{Condens.}$ Matter Mater. Phys. 2010, 81 (2), 020201.

(11) Goldman, A.; Kong, T.; Kreyssig, A.; Jesche, A.; Ramazanoglu, M.; Dennis, K.; Bud'ko, S.; Canfield, P. A family of binary magnetic icosahedral quasicrystals based on rare earths and cadmium. Nat. Mater. 2013, $12(8), 714-8$.

(12) Canfield, P. C. New materials physics. Rep. Prog. Phys. 2020, 83 (1), 016501.

(13) Reichmann, T. L.; Ipser, H. Reinvestigation of the Cd-Gd phase diagram. J. Alloys Compd. 2014, 617, 292-301.

(14) Canfield, P.; Kong, T.; Kaluarachchi, U.; Jo, N. H. Use of frit-disc crucibles for routine and exploratory solution growth of single crystalline samples. Philos. Mag. 2016, 96 (1), 84-92.

(15) Degen, T.; Sadki, M.; Bron, E.; König, U.; Nénert, G. HighScore suite, 3.0.5; Volume 29, 2014.

(16) Labib, F.; Fujita, N.; Ohhashi, S.; Tsai, A.-P. Icosahedral quasicrystals and their cubic approximants in the Cd-Mg-RE (RE= Y, $\mathrm{Sm}, \mathrm{Gd}, \mathrm{Tb}, \mathrm{Dy}, \mathrm{Ho}, \mathrm{Er}, \mathrm{Tm}$ ) systems. J. Alloys Compd. 2020, 822, 153541 .

(17) Goldman, A.; Kelton, R. Quasicrystals and crystalline approximants. Rev. Mod. Phys. 1993, 65 (1), 213-230.

(18) Wang, P.; Stadnik, Z. M.; Al-Qadi, K.; Przewoźnik, J. A comparative study of the magnetic properties of the $1 / 1$ approximant Ag50In36Gd14 and the icosahedral quasicrystal Ag50In36Gd14. J. Phys.: Condens. Matter 2009, 21 (43), 436007.

(19) Larson, A. C.; Cromer, D. T. The crystal structure of YCd6. Acta Crystallogr., Sect. B: Struct. Crystallogr. Cryst. Chem. 1971, 27 (10), $1875-1879$.

(20) Pay Gómez, C.; Lidin, S. Comparative structural study of the disordered MCd6 quasicrystal approximants. Phys. Rev. B 2003, 68 (2), 024203.

(21) Tsai, A.-P. Discovery of stable icosahedral quasicrystals: progress in understanding structure and properties. Chem. Soc. Rev. 2013, 42 (12), 5352-5365.

(22) Ishimasa, T.; Tanaka, Y.; Kashimoto, S. Icosahedral quasicrystal and $1 / 1$ cubic approximant in Au-Al-Yb alloys. Philos. Mag. 2011, 91 (33), 4218-4229. 